\title{
Regeneration of injured tissue: stem cell dynamics at interplay with mTORC1
}

\author{
Mario Mikula \\ Center for Pathobiochemistry and Genetics, Institute of Medical Genetics, Medical University of Vienna, Vienna, Austria \\ Correspondence to: Dr. Mario Mikula. Center for Pathobiochemistry and Genetics, Institute of Medical Genetics, Medical University of Vienna, \\ Währingerstrasse 10, 1090 Vienna, Austria. Email: mario.mikula@meduniwien.ac.at. \\ Comment on: Haller S, Kapuria S, Riley RR, et al. mTORC1 Activation during Repeated Regeneration Impairs Somatic Stem Cell Maintenance. Cell \\ Stem Cell 2017;21:806-18.e5.
}

Received: 10 July 2018; Accepted: 06 August 2018; Published: 30 August 2018.

doi: $10.21037 /$ sci.2018.08.01

View this article at: http://dx.doi.org/10.21037/sci.2018.08.01

The process of aging and whether it can be slowed down have raised questions which have troubled mankind since its early beginnings. Only recently we have developed the tools to further investigate the complex issue of aging. It has become clear that certain hallmarks can be attributed to the aging process which include genomic instability, telomere attrition, epigenetic alterations, loss of proteostasis, deregulated nutrient sensing, mitochondrial dysfunction, cellular senescence, altered intercellular communication and stem cell exhaustion (1). Especially the latter has raised increasing attention due to the importance of stem cells for tissue regeneration. There is great hope for rejuvenating aged tissue by the prevention of continuous stem cell loss or by reestablishing the initial stem cell amount.

In an article published recently in Cell Stem Cell, a research group lead by Heinrich Jasper, demonstrated that stem cells transiently activate mechanistic target of rapamycin complex 1 (mTORC1) during tissue regeneration and that chronic mTORC1 signaling lead to stem cell loss in the tissue under investigation (2). Tissue homeostasis is obtained by balanced proliferation and apoptosis of somatic cells. During repeated tissue injury and repair somatic stem cells are needed to enable full regeneration. The effect of mTOR modulation on barrier epithelial tissue homeostasis like in the fly intestine and in the mouse tracheal epithelium has been investigated by Haller et al. (2). To induce repeated epithelial tissue damage and activation of stem cells, either drosophila was orally infected with Erwinia carotovora, or mice were exposed to breathing air containing $500 \mathrm{ppm}$ of $\mathrm{SO}_{2}$. This treatment resulted in mTORC1 activation evidenced by 4EBP and RPS6 phosphorylation, respectively. Repeated damaging reduced the amount of tissue specific stem cells and importantly loss of TSC1, a repressor of mTORC1 activity, also enhanced this effect. Furthermore, the authors showed that rapamycin treatment of mice could prevent the loss of tracheal stem cells after repeated injury. The inhibition of chronic mTORC1 by treatment with rapamycin and its analogs is depicted by a schematic in Figure 1. A genetic model for mTORC1 inhibition is the deletion of Raptor. Likewise, authors could show that Raptor knockout mice, compared to controls, maintained a higher amount of muscle stem cells in aged animals. Finally, tracheal epithelial stem cells loss due to aging could be inhibited by chronic rapamycin treatment. Taken together these findings highlight mTORC1 as a central node in stem cell activation. Additionally, chronic mTORC1 signaling is also causatively involved in stem cell exhaustion over time. Therefore, potentially reducing tissue specific regeneration and repair. To further explore this conundrum, it is important to recapitulate what is already known on mTORC1 mode of action in distinct cell types and how this could be used for the benefit of human patients.

mTOR is an evolutionary conserved protein kinase, which is present in at least two protein complexes, namely mTORC1 and mTORC2 (3). Its function is to integrate environmental signals like growth factors and nutrients as well as intracellular signals like oxygen content and amino acid availability (4). mTORC1 controls cell cycle progression, protein and lipid synthesis (5). Activation of this pathway for example promotes regeneration of axons 


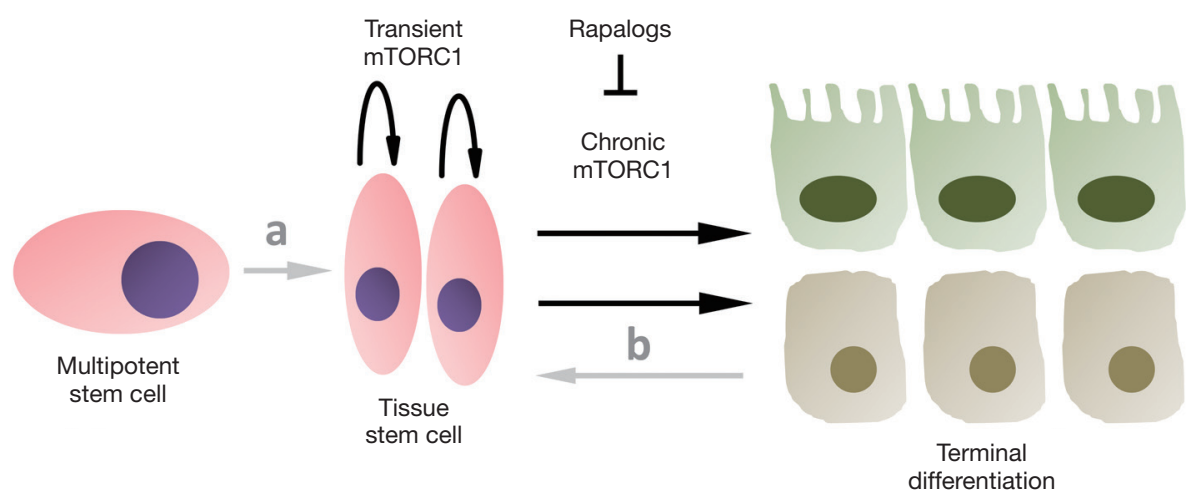

Figure 1 Tissue stem cell dynamics. Schematic flux model for regulation of tissue stem cell amounts. Transient mTORC1 activation is observed in tissue stem cells during regeneration. Chronic mTORC1 activation leads to terminal differentiation and therefore stem cell loss. Blocking chronic mTORC1 by rapamycin and its analogs (rapalogs) limits stem cell loss. Potential alternative pathways to maintain the tissue stem cell pool are (a) multipotent stem cells give rise to novel tissue stem cells, sometimes referred to plasticity of stem cells and (b) differentiated cells are reprogrammed to become multipotent progenitor cells again. Arrows indicate transition between cell types.

in the adult central nervous system (6), or enables early steps during Schwann cell differentiation from a specific stem cell population (7), both are examples where high lipid synthesis is needed. Inhibition of mTORC1 by raptor depletion or by rapamycin treatment inhibits protein translation and activates autophagy in cells (5). Therefore, repression of mTORC1 plays an important housekeeping role in energy metabolism (8), exemplary inhibition of mTORC1 enhanced the chondrogenic phenotype, which enables survival under extreme starvation conditions (9). Importantly mTORC1 needs to be tightly controlled in adult stem cells as it has been recently shown that ERK signaling prevents hematopoietic stem cell exhaustion by counterbalancing mTORC1 activity (10).

Despite the characterization of mTORC1 in tissue stem cell homeostasis the contribution of mTOR to alternative developmental pathways leading to tissue stem cell generation is largely unknown. In principal tissue independent multipotent stem cells as well as endogenous reprogramming of differentiated somatic cells can serve as sources for achieving tissue homeostasis. The two alternative pathways ( $\mathrm{a}$ and $\mathrm{b}$ ) for maintaining tissue stem cells are indicated in Figure 1. Multipotent stem cells are present in adult organism as hematopoietic stem cells and mesenchymal stem cells (11). Albeit both cellular pools are well known to regenerate somatic cells within their respective hierarchy there are also reports of so called stem cell plasticity. For mesenchymal stem cells non-linear transitions between cell populations can be observed in vivo and transplantation of hematopoietic stem cells to irradiated hosts demonstrated differentiation into epithelial cells of the liver, lung, gastro-intestinal tract and skin $(12,13)$. When hematopoietic stem cell grafting was used to regenerate non-hematopoietic tissue significant tissue injury was necessary to achieve a limited amount of cellular contribution as shown in examples of muscle tissue and liver regeneration $(14,15)$. Overall plasticity of adult stem cells is still often debated and most beneficial effects are now attributed to transient cell incorporation and paracrine signaling effects (16).

Recently, increasing attention is paid to the cell endogenous potential for regeneration where somatic cells de-differentiate and reach a multipotent state. The potential of differentiated cells to become bipotent progenitors has for example been shown in adult mouse hepatocytes (17). Multipotent progenitors have now been generated form neuronal cells where injury induced reprogramming was induced (18). It will be interesting to see whether other cells are also capable of this type of regeneration and whether mTORC1 inhibition also plays a role in this process.

Human mTORC1 can be inhibited by simple calorie restriction without malnutrition (19). Still most often pharmacologic mTOR inhibitors are used and a life prolonging effect of these inhibitors has been observed in a wide range of organisms (20-22). As the study by Haller et al. showed that rapamycin treatment rescues the loss of tissue stem cells also in aged animals it is interesting to note that feeding rapamycin to mice late in life also extended their lifespan (23). Pharmacologic mTOR inhibitors for human use are well known due to their wide clinical 
application. Thus raising the possibility that rejuvenation of aged tissue in humans is possible. However, their use has raised awareness in the multiple side effects which were induced in patients. Most frequently immunosuppression, interstitial pneumonitis, hyperglycemia and dyslipidemia were detected (24). This has to be taken into account when treatment regiments are designed in order to inhibit the aging process. A possible way to circumvent unwanted effects would be alternating dosing. By doing so mTOR activity would be enabled during the regeneration process, but subsequently when homeostasis is reached, mTOR inhibition would be used to replenish the tissue stem cell pool. Thereby the paradox of mTORC1 function during stem cell maintenance as well as terminal differentiation can also be resolved. In detail, short periods of inhibition would block chronic signaling and therefore prevent stem cell exhaustion, while alternating periods without inhibition would enable stem cell activation and generation of sufficient amounts of somatic tissue cells. Stepwise blocking of mTOR, by yet to establish protocols, might be the key for converting aged tissue into juvenile-like tissue with a minimum of detrimental effects on the patient.

\section{Acknowledgements}

Funding: M Mikula is supported by grants from the NFB (LS14-007).

\section{Footnote}

Conflicts of Interest: The author has no conflicts of interest to declare.

\section{References}

1. López-Otín C, Blasco MA, Partridge L, et al. The hallmarks of aging. Cell 2013;153:1194-217.

2. Haller S, Kapuria S, Riley RR, et al. mTORC1 Activation during Repeated Regeneration Impairs Somatic Stem Cell Maintenance. Cell Stem Cell 2017;21:806-18.e5.

3. Laplante $M$, Sabatini DM. mTOR signaling at a glance. J Cell Sci 2009;122:3589-94.

4. Laplante M, Sabatini DM. mTOR signaling in growth control and disease. Cell 2012;149:274-93.

5. Laplante $M$, Sabatini DM. An emerging role of mTOR in lipid biosynthesis. Curr. Biol 2009;19:R1046-52.

6. Park KK, Liu K, Hu Y, et al. Promoting axon regeneration in the adult CNS by modulation of the PTEN/mTOR pathway. Science 2008;322:963-6.

7. Preitschopf A, Li K, Schörghofer D, et al. mTORC1 is essential for early steps during Schwann cell differentiation of amniotic fluid stem cells and regulates lipogenic gene expression. PLoS One 2014;9:e107004.

8. Rabinowitz JD, White E. Autophagy and metabolism. Science 2010;330:1344-8.

9. Preitschopf A, Schörghofer D, Kinslechner K, et al. Rapamycin-Induced Hypoxia Inducible Factor 2A Is Essential for Chondrogenic Differentiation of Amniotic Fluid Stem Cells. Stem Cells Transl Med 2016;5:580-90.

10. Baumgartner C, Toifl S, Farlik M, et al. An ERKDependent Feedback Mechanism Prevents Hematopoietic Stem Cell Exhaustion. Cell Stem Cell 2018;22:879-92.e6.

11. Friedenstein AJ, Petrakova KV, Kurolesova AI, et al. Heterotopic of bone marrow. Analysis of precursor cells for osteogenic and hematopoietic tissues. Transplantation 1968;6:230-47.

12. Krause DS, Theise ND, Collector MI, et al. Multi-organ, multi-lineage engraftment by a single bone marrowderived stem cell. Cell 2001;105:369-77.

13. Theise ND, Henegariu O, Grove J, et al. Radiation pneumonitis in mice: a severe injury model for pneumocyte engraftment from bone marrow. Exp Hematol 2002;30:1333-8.

14. Corbel SY, Lee A, Yi L, et al. Contribution of hematopoietic stem cells to skeletal muscle. Nat Med 2003;9:1528-32.

15. Tsolaki E, Athanasiou E, Gounari E, et al. Hematopoietic stem cells and liver regeneration: differentially acting hematopoietic stem cell mobilization agents reverse induced chronic liver injury. Blood Cells Mol Dis 2014;53:124-32.

16. Kfoury Y, Scadden DT. Mesenchymal cell contributions to the stem cell niche. Cell Stem Cell 2015;16:239-53.

17. Mikula M, Fuchs E, Huber H, et al. Immortalized p19ARF null hepatocytes restore liver injury and generate hepatic progenitors after transplantation. Hepatology 2004;39:628-34.

18. Lin B, Coleman JH, Peterson JN, et al. Injury induces endogenous reprogramming and dedifferentiation of neuronal progenitors to multipotency. Cell Stem Cell 2017;21:761-74.e5.

19. Weichhart T. mTOR as Regulator of Lifespan, Aging, and Cellular Senescence: A Mini-Review. Gerontology 2018;64:127-34.

20. Miller RA, Harrison DE, Astle CM, et al. Rapamycin, but not resveratrol or simvastatin, extends life span of 
genetically heterogeneous mice. J Gerontol A Biol Sci Med Sci 2011;66:191-201.

21. Bjedov I, Toivonen JM, Kerr F, et al. Mechanisms of life span extension by rapamycin in the fruit fly Drosophila melanogaster. Cell Metab 2010;11:35-46.

22. Markofski MM, Dickinson JM, Drummond MJ, et al. Effect of age on basal muscle protein synthesis and

doi: $10.21037 /$ sci.2018.08.01

Cite this article as: Mikula M. Regeneration of injured tissue: stem cell dynamics at interplay with mTORC1. Stem Cell Investig 2018;5:27.
mTORC1 signaling in a large cohort of young and older men and women. Exp Gerontol 2015;65:1-7.

23. Harrison DE, Strong R, Sharp ZD, et al. Rapamycin fed late in life extends lifespan in genetically heterogeneous mice. Nature 2009;460:392-5.

24. Pallet N, Legendre C. Adverse events associated with mTOR inhibitors. Expert Opin Drug Saf 2013;12:177-86. 